\title{
Motivation and Social Skills in Nursing Students Compared to Physical Education Students
}

\section{Motivación y Habilidades Sociales en Estudiantes de Enfermería en comparación con Estudiantes de Educación Física}

\author{
Lionel Sánchez-Bolívar ${ }^{1}$, Sergio Escalante-González ${ }^{1 *}$, Asunción Martínez-Martínez ${ }^{1}$ \\ ${ }^{1}$ Departamento de Métodos de Investigación y Diagnóstico en Educación, Universidad de Granada, \\ Granada, España. \\ * Correspondence: Sergio Escalante-González; sescalante@correo.ugr.es
}

\begin{abstract}
One of the relevant factors that explain human behavior, in the acts that develops in daily life, is motivation. The aim of this study was to determinate the level of motivation and social skills in nursing degree student and compare with the level of motivation of physical education students. A descriptive and correlational study, with a cross-sectional design was used in this research $(n=101$; $22.03 \pm 5.8$ years old), using the Spanish version of the Situational Motivation Scale (SIMS). It can be highlighted that there is a predominantly female student population in this campus, with a higher motivational level. The predominant religion is the Christian/Catholic, counting with mean values in identified regulation superior to those of the rest. Regarding social skills, thinking (optimistic or pessimistic), empathy and assertiveness are the skills that influence the degree of motivation of students. Regarding the level of motivation, Nursing students show a medium-high level and Physical Education, a high level. Motivation is intrinsic in nursing students and more extrinsic in Physical Education students, although there are also high levels of intrinsic motivation.
\end{abstract}

\section{KEYWORDS}

Motivation; Nursing; Physical education; Social skills; University students 


\section{RESUMEN}

Uno de los factores relevantes que explican el comportamiento humano, en los actos que se desarrollan en la vida diaria, es la motivación. El objetivo de este estudio fue determinar el nivel de motivación y habilidades sociales en estudiantes de enfermería y compararlo con la motivación de estudiantes universitarios de educación física. Para ello, se realizó un estudio descriptivo y correlacional, con un diseño transversal, en $(\mathrm{n}=101 ; 22.03+5.8$ años), utilizando la Escala de Motivación Situacional (SIMS). Se puede resaltar que hay una población estudiantil predominantemente femenina, con un mayor nivel de motivación, hecho que se repite en estudiantes de Educación Física. La religión predominante es la cristiana/católica, contando con valores medios en una regulación identificada superior a la del resto. El pensamiento, la empatía y la asertividad son las habilidades que influyen en el grado de motivación de los estudiantes. Existe una relación negativa entre la motivación intrínseca y la pasividad y entre la motivación intrínseca y la agresividad. En cuanto al nivel de motivación, el alumnado de Enfermería manifiesta un nivel medioalto y el de Educación Física, un nivel alto. La motivación es intrínseca en estudiantes de enfermería y más extrínseca en el alumnado de Educación Física, aunque también, existen altos niveles de motivación intrínseca.

\section{PALABRAS CLAVE}

Motivación; Enfermería, Educación física; Habilidades sociales; Estudiantes universitarios

\section{INTRODUCTION}

University stage is a crucial period in human life due to the convergence of psychologically stressful situations for students. At this stage, students begin to regulate and assess their situation between academic achievement and their professional/employment future in the short/medium term.

In this sense, motivation is a psychological parameter, self-regulated and self-determined which will directly influence on academic and professional achievement, favoring or hindering their achievement, based on a high or low motivational level (Ryan \& Deci, 2017).

In relation to motivation, social skills such as empathy, leadership, emotional control, assertiveness, proactivity, etc., help to motivate to achieve goals and accept criticism (TapiaGutiérrez \& Cubo-Delgado, 2017). A low level of social ability leads to a feeling of social incompetence and, therefore, to the lack of motivation of the person, while a socially skilled person, that is, with a high level of development of social skills, feels socially competent (therefore, 
motivated), creating an autofeedback situation, encouraging him to continue developing his skills in a motivated way (Van der Stouwe et al., 2018).

When it comes to assessing student motivation, studies and research have focused on two aspects: the educational stage and the area of knowledge in which they have been carried out.

Depending on the area of knowledge, the students will experience variations in the level and type of motivation depending of the nature of each area. In the linguistic area, being more theoretical, there is a lower level of motivation and it is more intrinsic than other areas (Dörnyei, 2018). In areas such as mathematics, there is a motivational decline in the female gender, caused by the sexual stereotype that presupposes that men have greater mathematical capacity than women, increasing their motivation towards language and literature and men towards mathematics and physical education (Fernández-Lasarte et al., 2019).

It has to be emphasized that the type and level of motivation is different at each stage of: Preschool, Primary Education, Secondary Education, Further Education and Higher Education.

In pre-school stage, the students have a very high level of motivation and closeness (and affectivity) with the teachers. Those with extrinsic motivation experience a decrease in conflict (reduction of tantrums) over time. Motivation, at this stage, is directly linked to the teacher's actions and to the reinforcement or punishment that the students receive from them (Bosman et al., 2018).

In primary education, motivation is related to the proposed objective and self-efficacy, which is why the extrinsic strength of motivation is intensified, reducing the closeness with the teacher. It is based on a motivation directed towards the prize and the avoidance of punishment. Likewise, the level of this is linked to the level of resilience, so a decrease in it would cause a decrease in the other (Kim et al., 2018).

In secondary education, due to the temporal coincidence with adolescence, the motivation will be related to the social self-regulation of the students. At this stage, sexual stereotypes are increased, establishing an extrinsic and integrative motivational pattern in the students due to the need to be part of the peer group and the fear of exclusion (Fernández-Lasarte et al., 2019).

When the motivation is evaluated in university students, the specific degree in which the student is enrolled must be considered. Therefore, the established studies may be in university students, grouping various degrees; or, it will deal with the measurement of motivation in students of a specific degree (motivation in university students of the degree in sociology, for example).

In this pre-adult youth stage, motivation is linked to self-regulation and self-efficacy, directly related to students' self-concept and self-esteem and their academic achievements (Meijer et al., 2018). 
In order to establish a relational pattern between social skills and students motivation when pursuing a degree, the present study of university students' motivation in the cross-border of Ceuta is proposed with two objectives. This research aim is describe and analyze the variables age, gender, religion, reason for enrollment and social skills, relate the aforementioned variables with students' motivation, and compare with the motivation level of the physical education students.

\section{METHODS}

\subsection{Subjects and design}

A nonexperimental, descriptive, exploratory, correlational and cross-sectional study was carried out on a sample of 101 university students from Ceuta, enrolled in Nursing Degree in the University of Granada, Campus of Ceuta. The participants answered voluntarily after receiving a detailed explanation of the objectives and nature of the study. Written informed consent was provided. The sample selected, through an intentional sampling, a sample of 101 university students, $77.2 \%$ women $(n=78)$ and $22.8 \%(n=23)$ men.

\subsection{Instruments}

The variables defined in this study and the instruments that have been used to measure them are: age; gender (depending on the case, man or woman); religion; (collected through an ad-hoc questionnaire with five options: a) Christian/Catholic, b) Jewish/Hebrew, c) Muslim, d) Hindu and e) others), reason for enrollment (collected through an ad-hoc questionnaire with eleven options: a) Family expectation, b) Preference, c) Vocation, d) Having a job title, e) Personal satisfaction, f) Opting for a better job category, g) Being unemployed, h) Increasing the curriculum, i ) Have more professional knowledge, j) Lack of options, and k) Other); Social Skills (collected using an ad-hoc Likert-scale, with ten items that refer to different social skills, distributed according to the polarized categories: Introvert-Extrovert, Passive-Active, Reactive-Proactive, Asocial-Sociable, ReservedCommunicative, Pessimistic-Optimistic, Indifferent-Empathetic, Aggressive-Assertive, SubmissiveDominant, Inflexible-Adaptive; and Motivation.

Motivation measurement was carried out by the Spanish version of the Situational Motivation Scale, created and validated by Guay et al. (2000), adapted to Spanish by Martín-Albo et al. (2009). It consists of 16 items, on a Likert scale from 1 to 7 (where 1 is equivalent to nothing, 2 to very little, 3 a little, 4 to moderately, 5 to sufficient, 6 to much and 7 to totally), which evaluates four dimensions: intrinsic motivation (evaluated by items 1, 5, 9 and 13), identified regulation (evaluated by items $2,6,10,14$ ), external regulation (evaluated by items 3, 7, 11, 15), and amotivation 
(evaluated by items 4, 8, 12 and 16). For this study, the scale yielded an internal correlation coefficient, measured by Cronbach's alpha, of $\alpha=0.703$.

\subsection{Data analysis}

The 25.0 version of IBM SPSS (Statistical Package for the Social Sciences) program was used to analyze the results obtained.

In a first part, the descriptive analysis of the data obtained from the questionnaire is carried out, using descriptive statistics such as frequency, percentage, arithmetic mean, and standard deviation. In a second part, the relationship between the variables is established using the Student's $t$ test for dichotomous variables and the one-way ANOVA for polytomous variables. To measure the degree of relationship between variables, the Pearson's correlation coefficient was used.

\section{RESULTS}

The data showed a mean age of 20.43 years, where the lowest age was 18 years and the highest was 54. Table 1 shows the sociodemographic profile of the study population. As can be seen, the majority population is female, representing $77.2 \%$ of the total $(n=78)$, while the male population, with 97 individuals, represents $22.8 \%(n=23)$. Regarding religion, the majority is Catholic/Christian $(n=75 ; 74.3 \%)$.

Table 1. Descriptive statistics

\begin{tabular}{cllll}
\hline & Gender & N (\%) & $\begin{array}{c}\text { Total } \\
(\% \text { Total })\end{array}$ \\
\hline \multirow{2}{*}{ Population } & Male & $23(22.8 \%)$ & \\
& Female & $78(77.2 \%)$ & $101(100 \%)$ \\
& Christian/ & Male & $18(24 \%)$ & \\
Catholic & Female & $57(76 \%)$ & \\
Religion & Muslim & Male & $2(25 \%)$ & $8(7.9 \%)$ \\
& & Female & $6(75 \%)$ & \\
& Other & Male & $3(16.7 \%)$ & $18(17.8 \%)$ \\
& & Female & $15(83.3 \%)$ & \\
\hline
\end{tabular}

As can be seen in the following table (Table 2), the main initial motivation (reason for enrollment), of the students enrolled in Nursing degree, is "Vocation" with $71.30 \%(n=72)$, continued 
by the "Preference" (understood as the most interesting option, for the students, which they could choose) with $18.80 \%(\mathrm{~N}=19)$.

Table 2. Descriptive statistics for Reason for enrolment and Social Skills

\begin{tabular}{|c|c|c|c|c|c|c|c|}
\hline \multicolumn{8}{|c|}{ Reason for Enrolment } \\
\hline & \multicolumn{2}{|c|}{ Gender } & \multirow{2}{*}{ Total } & & \multicolumn{2}{|c|}{ Gender } & \multirow{2}{*}{ Total } \\
\hline & $\mathbf{M}$ & $\mathbf{F}$ & & & $\mathbf{M}$ & $\mathbf{F}$ & \\
\hline $\begin{array}{l}\text { Family } \\
\text { expectation }\end{array}$ & $0(0 \%)$ & $1(1 \%)$ & $1(1 \%)$ & $\begin{array}{l}\text { Opting for a } \\
\text { better job } \\
\text { category }\end{array}$ & $2(2 \%)$ & $0(0 \%)$ & $2(2 \%)$ \\
\hline Preference & $\begin{array}{c}14 \\
(13.9 \%)\end{array}$ & $5(5 \%)$ & $\begin{array}{c}19 \\
(18.8 \%)\end{array}$ & $\begin{array}{l}\text { Increasing } \\
\text { the } \\
\text { curriculum }\end{array}$ & $0(0 \%)$ & $0(0 \%)$ & $0(0 \%)$ \\
\hline Vocation & $\begin{array}{c}58 \\
(57.4 \%)\end{array}$ & $\begin{array}{c}14 \\
(13.9 \%)\end{array}$ & $\begin{array}{c}72 \\
(71.3 \%)\end{array}$ & $\begin{array}{l}\text { Have more } \\
\text { professional } \\
\text { knowledge }\end{array}$ & $0(0 \%)$ & $0(0 \%)$ & $0(0 \%)$ \\
\hline $\begin{array}{l}\text { Having a job } \\
\text { title }\end{array}$ & $0(0 \%)$ & $0(0 \%)$ & $0(0 \%)$ & $\begin{array}{l}\text { Lack of } \\
\text { options }\end{array}$ & $0(0 \%)$ & $1(1 \%)$ & $1(1 \%)$ \\
\hline $\begin{array}{l}\text { Personal } \\
\text { satisfaction }\end{array}$ & $3(3 \%)$ & $2(2 \%)$ & $5(5 \%)$ & Other & $1(1 \%)$ & $0(0 \%)$ & $1(1 \%)$ \\
\hline \multicolumn{8}{|c|}{ Social Skills } \\
\hline & \multicolumn{2}{|c|}{ Gender } & \multirow{2}{*}{ Total } & & \multicolumn{2}{|c|}{ Gender } & \\
\hline & $\mathbf{M}$ & $\mathbf{F}$ & & & M & $\mathbf{F}$ & Total \\
\hline Introvert & $\begin{array}{c}26 \\
(25.7 \%)\end{array}$ & $5(5 \%)$ & $\begin{array}{c}31 \\
(30.7 \%)\end{array}$ & Pessimistic & $\begin{array}{c}32 \\
(31.7 \%)\end{array}$ & $\begin{array}{c}7 \\
(6.9 \%)\end{array}$ & $\begin{array}{c}39 \\
(38.6 \%)\end{array}$ \\
\hline Extrovert & $\begin{array}{c}52 \\
(51.5 \%)\end{array}$ & $\begin{array}{c}18 \\
(17.8 \%)\end{array}$ & $\begin{array}{c}70 \\
(69.3 \%)\end{array}$ & Optimistic & $\begin{array}{c}46 \\
(45.5 \%)\end{array}$ & $\begin{array}{c}16 \\
(15.8 \%)\end{array}$ & $\begin{array}{c}62 \\
(61.4 \%)\end{array}$ \\
\hline Passive & $\begin{array}{c}17 \\
(16.8 \%)\end{array}$ & $3(3 \%)$ & $\begin{array}{c}20 \\
(19.8 \%)\end{array}$ & Indifferent & $\begin{array}{c}7 \\
(6.9 \%)\end{array}$ & $1(1 \%)$ & $\begin{array}{c}8 \\
(7.9 \%)\end{array}$ \\
\hline Active & $\begin{array}{c}61 \\
(60.4 \%)\end{array}$ & $\begin{array}{c}20 \\
(19.8 \%)\end{array}$ & $\begin{array}{c}81 \\
(80.2 \%)\end{array}$ & Empathetic & $\begin{array}{c}71 \\
(70.3 \%)\end{array}$ & $\begin{array}{c}22 \\
(21.8 \%)\end{array}$ & $\begin{array}{c}93 \\
(92.1 \%)\end{array}$ \\
\hline Reactive & $\begin{array}{c}23 \\
(22.8 \%)\end{array}$ & $\begin{array}{c}6 \\
(5.9 \%)\end{array}$ & $\begin{array}{c}29 \\
(28.7 \%)\end{array}$ & Aggressive & $\begin{array}{c}20 \\
(19.8 \%)\end{array}$ & $5(5 \%)$ & $\begin{array}{c}25 \\
(24.8 \%)\end{array}$ \\
\hline Proactive & $\begin{array}{c}55 \\
(54.5 \%)\end{array}$ & $\begin{array}{c}17 \\
(16.8 \%)\end{array}$ & $\begin{array}{c}72 \\
(71.3 \%)\end{array}$ & Assertive & $\begin{array}{c}58 \\
(57.4 \%)\end{array}$ & $\begin{array}{c}18 \\
(17.8 \%)\end{array}$ & $\begin{array}{c}76 \\
(75.2 \%)\end{array}$ \\
\hline Asocial & $\begin{array}{c}13 \\
(12.9 \%)\end{array}$ & $1(1 \%)$ & $\begin{array}{c}14 \\
(13.9 \%)\end{array}$ & Submissive & $\begin{array}{c}33 \\
(32.7 \%)\end{array}$ & $\begin{array}{c}7 \\
(6.9 \%)\end{array}$ & $\begin{array}{c}40 \\
(39.6 \%)\end{array}$ \\
\hline Social & $\begin{array}{c}65 \\
(64.4 \%)\end{array}$ & $\begin{array}{c}22 \\
(21.8 \%)\end{array}$ & $\begin{array}{c}87 \\
886,1 \%)\end{array}$ & Dominant & $\begin{array}{c}45 \\
(44.6 \%)\end{array}$ & $\begin{array}{c}16 \\
(15.8 \%)\end{array}$ & $\begin{array}{c}61 \\
(60.4 \%)\end{array}$ \\
\hline Reserved & $\begin{array}{c}22 \\
(21.8 \%)\end{array}$ & $4(4 \%)$ & $\begin{array}{c}26 \\
(25.7 \%)\end{array}$ & Inflexible & $4(4 \%)$ & $1(1 \%)$ & $5(5 \%)$ \\
\hline Communicative & $\begin{array}{c}56 \\
(55.4 \%)\end{array}$ & $\begin{array}{c}19 \\
(18.8 \%)\end{array}$ & $\begin{array}{c}75 \\
(74.3 \%)\end{array}$ & Adaptive & $\begin{array}{c}74 \\
(73.3 \%)\end{array}$ & $\begin{array}{c}22 \\
(21.8 \%)\end{array}$ & $\begin{array}{c}96 \\
(95 \%)\end{array}$ \\
\hline
\end{tabular}

Regarding social skills, students show a medium-high level of social skills, highlighting "adaptability" ( $n=96 ; 95 \%)$ and "empathy" ( $n=93 ; 92.1 \%)$, as predominant skills in the studied 
population. In the opposite pole, in terms of attitude, 38.6\% ( $n=39)$ considered themselves pessimistic. Likewise, $60.4 \%(n=61)$ of the population is considered "dominant" compared to $39.6 \%$ $(n=40)$ who consider themselves "submissive".

Regarding the dimensions of the scale, as observed in Table 3, the dimension with higher mean values is the "identified regulation" $(\mathrm{M}=6.13$; $\mathrm{SD}=1.42)$, while the dimension with lower mean values is the "amotivation" $(\mathrm{M}=1.81 ; \mathrm{SD}=1.10)$.

Table 3. Average values of the dimensions of the SIMS and ANOVA with gender and religion

\begin{tabular}{|c|c|c|c|c|c|}
\hline & & Minimum & Maximum & $\mathbf{M}$ & SD \\
\hline \multicolumn{2}{|c|}{ Intrinsic Motivation } & 1.00 & 7.00 & 5.11 & 1.42 \\
\hline \multicolumn{2}{|c|}{ Identified Regulation } & 2.00 & 7.00 & 6.13 & 0.93 \\
\hline \multicolumn{2}{|c|}{ External Regulation } & 1.00 & 7.00 & 3.50 & 1.44 \\
\hline \multicolumn{2}{|c|}{ Amotivation } & 1.00 & 5.75 & 1.81 & 1.10 \\
\hline \multicolumn{6}{|c|}{ Relationship between motivation and gender } \\
\hline & & M & SD & $\mathbf{F}$ & $\mathbf{p}$ \\
\hline Intrinsic & Female & 5.58 & 1.29 & \multirow{2}{*}{1.055} & \multirow{2}{*}{0.160} \\
\hline Motivation & Male & 5.16 & 1.06 & & \\
\hline Identified & Female & 6.24 & 0.90 & \multirow{2}{*}{0.014} & \multirow{2}{*}{0.243} \\
\hline Regulation & Male & 5.98 & 1.06 & & \\
\hline External & Female & 3.31 & 1.26 & \multirow{2}{*}{0.826} & \multirow{2}{*}{0.470} \\
\hline Regulation & Male & 3.53 & 1.48 & & \\
\hline \multirow{2}{*}{ Amotivation } & Female & 1.45 & 0.77 & \multirow{2}{*}{5.274} & \multirow{2}{*}{0.045} \\
\hline & Male & 1.88 & 1.23 & & \\
\hline \multicolumn{6}{|c|}{ Relationship between motivation and religion } \\
\hline \multirow{4}{*}{$\begin{array}{l}\text { Intrinsic } \\
\text { Motivation }\end{array}$} & & $\mathbf{M}$ & SD & $\mathbf{F}$ & $\mathbf{p}$ \\
\hline & Christian/Catholic & 5.62 & 1.15 & \multirow{3}{*}{1.832} & \multirow{3}{*}{0.166} \\
\hline & Muslim & 5.03 & 1.21 & & \\
\hline & Other & 5.11 & 1.55 & & \\
\hline \multirow{3}{*}{$\begin{array}{l}\text { Identified } \\
\text { Regulation }\end{array}$} & Christian/Catholic & 6.22 & 0.93 & \multirow{3}{*}{0.303} & \multirow{3}{*}{0.739} \\
\hline & Muslim & 6.09 & 0.76 & & \\
\hline & Other & 6.04 & 1.08 & & \\
\hline \multirow{3}{*}{$\begin{array}{l}\text { External } \\
\text { Regulation }\end{array}$} & Christian/Catholic & 3.44 & 1.38 & \multirow{3}{*}{0.940} & \multirow{3}{*}{0.394} \\
\hline & Muslim & 2.78 & 0.60 & & \\
\hline & Other & 3.29 & 1.18 & & \\
\hline \multirow{3}{*}{ Amotivation } & Christian/Catholic & 1.61 & 0.95 & \multirow{3}{*}{0.820} & \multirow{3}{*}{0.443} \\
\hline & Muslim & 1.22 & 0.25 & & \\
\hline & Other & 1.44 & 0.90 & & \\
\hline
\end{tabular}

In relation to "gender", as can be seen in Table 4, there are no statistically significant differences $(p>0.05)$ between the "gender" of the student body and the "intrinsic motivation" $(p=$ 
$0.160)$, the same it happens with the "identified regulation" ( $p=0.243)$ and with the "external regulation" ( $p=0.470)$.

On the other hand, statistically significant differences $(p<0.05)$ have been found in the relationship between the "gender" of the student body and the "amotivation" ( $p=0.045)$, in which we find the higher mean values in the male gender $(\mathrm{M}=1.88$; $\mathrm{SD}=1.23)$ and the lower ones in the female $(M=1.45 ; \mathrm{SD}=0.77)$.

Regarding religion (Table 5), no statistically significant differences ( $p>0.05$ ) have been identified between this and the four dimensions that make up the scale.

Regarding the relationship between "social skills" and students motivation (table 4), statistically significant differences have been found between "optimistic / pessimistic" thinking and "intrinsic motivation" ( $p=0.024)$, the upper mean values are found in the "optimistic" students $(\mathrm{M}=$ $5.71 ; \mathrm{SD}=1.01)$ and the lower ones in the "pessimistic" $(\mathrm{M}=5.13 ; \mathrm{SD}=1.50)$.

On the other hand, there are statistically significant differences $(p<0.05)$ between "pessimistic / optimistic" thinking and "external regulation" ( $p=0.030)$, found the highest values in "pessimistic" students $(\mathrm{M}=3.71 ; \mathrm{SD}=1.49)$ and the lowest in the "optimist" $(\mathrm{M}=3.14 ; \mathrm{SD}=1.13)$.

Table 4. Relationship between social skills and motivation

\begin{tabular}{|c|c|c|c|c|c|c|c|c|c|c|c|c|c|c|c|}
\hline \multicolumn{2}{|c|}{ Attitude } & \multirow{3}{*}{$\begin{array}{c}\mathbf{M} \\
5.63 \\
5.42\end{array}$} & \multirow{3}{*}{$\begin{array}{l}\text { SD } \\
1.06 \\
1.32\end{array}$} & \multirow{3}{*}{$\frac{\mathbf{F}}{0.352}$} & \multirow{3}{*}{$\begin{array}{l}p \\
.404\end{array}$} & \multirow{3}{*}{$\begin{array}{l}\text { Activity } \\
\text { PAS } \\
\text { ACT }\end{array}$} & \multirow{3}{*}{$\begin{array}{c}\mathbf{M} \\
5.55 \\
5.47\end{array}$} & \multirow{3}{*}{$\begin{array}{l}\text { SD } \\
1.00 \\
1.30\end{array}$} & \multirow{3}{*}{$\frac{\mathbf{F}}{1.274}$} & \multirow{3}{*}{$\frac{p}{0.764}$} & \multirow{3}{*}{$\begin{array}{l}\text { Communication } \\
\text { RES } \\
\text { COM }\end{array}$} & \multirow{3}{*}{$\begin{array}{c}\mathbf{M} \\
5.61 \\
5.44\end{array}$} & \multirow{3}{*}{$\begin{array}{c}\text { SD } \\
0.93 \\
1.34\end{array}$} & \multirow{3}{*}{$\frac{\mathbf{F}}{2.126}$} & \multirow{3}{*}{$\frac{p}{0.499}$} \\
\hline & INT & & & & & & & & & & & & & & \\
\hline MI & EXT & & & & & & & & & & & & & & \\
\hline \multirow{2}{*}{ RI } & INT & 6.19 & 0.86 & \multirow{2}{*}{0.098} & \multirow{2}{*}{.924} & PAS & 6.30 & 0.73 & \multirow{2}{*}{0.250} & \multirow{2}{*}{0.455} & RES & 6.30 & 0.67 & \multirow{2}{*}{2.031} & \multirow{2}{*}{0.372} \\
\hline & EXT & 6.18 & 0.98 & & & ACT & 6.15 & 0.99 & & & $\mathrm{COM}$ & 6.14 & 1.02 & & \\
\hline \multirow{2}{*}{$\mathrm{RE}$} & INT & 3.38 & 1.43 & \multirow{2}{*}{1.908} & \multirow{2}{*}{.923} & PAS & 3.41 & 1.30 & \multirow{2}{*}{0.012} & \multirow{2}{*}{0.839} & RES & 3.20 & 1.32 & \multirow{2}{*}{0.030} & 0485 \\
\hline & EXT & 3.35 & 1.25 & & & ACT & 3.35 & 1.31 & & & $\mathrm{COM}$ & 3.41 & 1.30 & & 0.485 \\
\hline AM & INT & 1.48 & 0.86 & 007 & 570 & PAS & 1.39 & 0.56 & 2007 & רכרים & RES & 1.56 & 0.90 & 0025 & 057 \\
\hline AM & EXT & 1.58 & 0.93 & 0.216 & $.5 / 9$ & $\mathrm{ACT}$ & 1.59 & 0.97 & 3.091 & 0.227 & $\mathrm{COM}$ & 1.55 & 0.91 & 0.035 & $0.95 /$ \\
\hline $\begin{array}{l}\text { Situa } \\
\text { Reac }\end{array}$ & $\begin{array}{l}\text { tional } \\
\text { tion }\end{array}$ & $\mathbf{M}$ & SD & $\mathbf{F}$ & $p$ & Sociability & $\mathbf{M}$ & SD & $\mathbf{F}$ & $p$ & Thinking & $\mathbf{M}$ & SD & $\mathbf{F}$ & $p$ \\
\hline 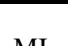 & $\mathrm{RE}$ & 5.28 & 1.31 & 0051 & 0220 & ASO & 5.45 & 1.00 & & & PES & 5.13 & 1.50 & & \\
\hline MI & PRO & 5.57 & 1.22 & 0.051 & 0.324 & SOC & 5.49 & 1.29 & 0.849 & 0.882 & OPT & 5.71 & 1.01 & 5.594 & 0.024 \\
\hline & $\mathrm{RE}$ & 6.05 & 1.05 & 0660 & 0440 & ASO & 6.13 & 0.74 & & & PES & 6.13 & 1.13 & & \\
\hline $\mathrm{RI}$ & PRO & 6.23 & 0.90 & 0.660 & 0.419 & SOC & 6.19 & 0.97 & 0.674 & 0.775 & OPT & 6.21 & 0.81 & 0.717 & 0.683 \\
\hline סחי & $\mathrm{RE}$ & 3.55 & 1.43 & & 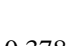 & ASO & 3.20 & 1.11 & & 078 & PES & 3.71 & 1.49 & & \\
\hline RE & PRO & 3.28 & 1.25 & 1.212 & 0.378 & SOC & 3.39 & 1.34 & 1.080 & 0.572 & OPT & 3.14 & 1.13 & 6.564 & 0.030 \\
\hline MM & $\mathrm{RE}$ & 1.58 & 0.87 & 000 & 084 & ASO & 1.46 & 0.78 & 080 & 0677 & PES & 1.69 & 1.04 & & 0230 \\
\hline AM & PRO & 1.54 & 0.92 & 0.001 & 0.841 & SOC & 1.56 & 0.93 & 0.800 & 0.674 & OPT & 1.46 & 0.08 & 3.091 & 0.239 \\
\hline Abili & & $\mathbf{M}$ & SD & $\mathbf{F}$ & $p$ & $\begin{array}{l}\text { Emotional } \\
\text { Reaction }\end{array}$ & $\mathbf{M}$ & SD & $\mathbf{F}$ & $p$ & Adaptivity & $\mathbf{M}$ & SD & $\mathbf{F}$ & $p$ \\
\hline 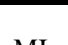 & IND & 5.09 & 1.36 & $0>2$ & 041 & AGR & 5.06 & 1.51 & 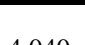 & $00 \Omega$ & INF & 5.45 & 1.16 & 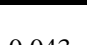 & م \\
\hline MI & EMP & 5.52 & 1.24 & 0.346 & 0.416 & ASE & 5.63 & 1.13 & 4.040 & 0.049 & $\mathrm{ADA}$ & 5.49 & 1.26 & 0.043 & 0.948 \\
\hline RI & IND & 6.34 & 0.52 & 1426 & 0413 & AGR & 6.05 & 1.21 & 2002 & & INF & 6.00 & 0.50 & 1028 & 0467 \\
\hline RI & EMP & 6.17 & 0.97 & 1.426 & 0.413 & ASE & 6.22 & 0.84 & 2.022 & 0.509 & ADA & 6.19 & 0.96 & 1.028 & 0.467 \\
\hline & IND & 4.03 & 1.82 & 3754 & 0300 & AGR & 3.50 & 1.40 & 1060 & 0556 & INF & 3.40 & 2.14 & & \\
\hline RE & EMP & 3.30 & 1.25 & 3.754 & 0.300 & ASE & 3.31 & 1.28 & 1.069 & 0.556 & $\mathrm{ADA}$ & 3.36 & 1.26 & 3.764 & 0.966 \\
\hline $\mathrm{AM}$ & IND & 1.56 & 0.94 & 0.074 & 0.968 & AGR & 1.74 & 1.20 & 4.749 & 0.227 & $\mathrm{INF}$ & 1.30 & 0.33 & 2.348 & 0.169 \\
\hline
\end{tabular}




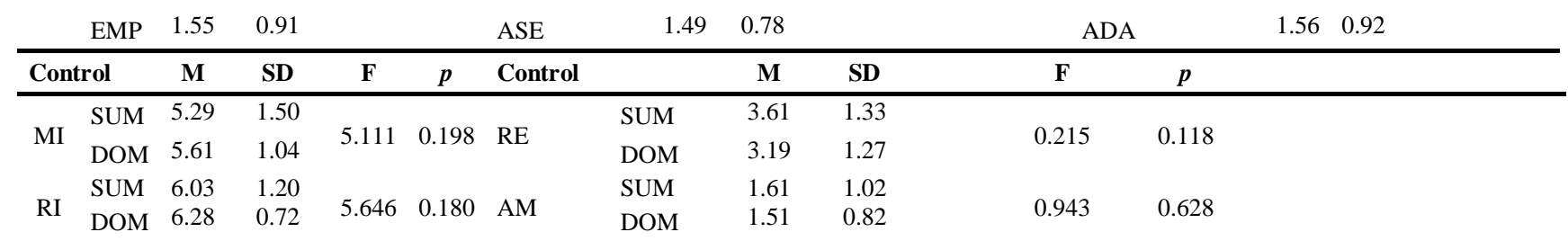

Note: Motivation; RI: Identified Regulation; RE: External Regulation; AM: Amotivation; INT: Introvert; EXT: Extrovert; PAS: Passive; ACT: Active; RES: Reserved; COM: Communicative; RE: Reactive; PRO: Proactive; ASO: Asocial; SOC; Sociable; OPT: Optimistic; PES: Pessimistic; IND: Indifferent; EMP: Empathetic; AGR: Aggressive; ASE: Assertive; INF: Inflexible; ADA: Adaptive; SUM: Submissive; DOM: Dominant.

On the other hand, there are statistically significant differences $(p<0.05)$ between the "aggressive / assertive" emotional reaction and the "intrinsic motivation" ( $p=0.049)$, in which the highest values are found in the "assertive" students $(\mathrm{M}=5.63 ; \mathrm{SD}=1.13)$ and the lowest in the “aggressive" ( $\mathrm{M}=5.06 ; \mathrm{SD}=1.51)$.

Finally, Table 5 reflects the matrix of correlations established between the variables. As can be seen, the "intrinsic motivation" is negatively correlated with the "external regulation" $(r=-0.006)$ and with the "amotivation" $(r=-0.249)$, being the correlation with the latter was significantly moderate. Regarding social skills, "intrinsic motivation" negatively correlates with "introversion" ( $r$ $=-0.077)$, with "passivity" $(\mathrm{r}=-0.026)$, with the lack of communicativeness $(\mathrm{r}=-0.057)$. On the other hand, it correlates positively with other social skills, and significantly with "optimism" $(r=$ $0.224)$ and with "assertiveness" ( $r=0.197)$.

Table 5. Correlations matrix

\begin{tabular}{|c|c|c|c|c|c|c|c|c|c|c|c|c|c|}
\hline & $\mathbf{R I}$ & RE & $\mathbf{A M}$ & IE & PA & $\mathbf{R P}$ & AS & $\mathbf{R C}$ & OP & INE & $\mathbf{A A}$ & SD & IA \\
\hline MI & $0.665^{\text {** }}$ & -0.006 & $-0.249^{*}$ & -0.077 & -0.026 & 0.103 & 0.013 & -0.057 & $0.224^{*}$ & 0.093 & $0.197^{*}$ & 0.129 & 0.006 \\
\hline RI & & 0.063 & $-0.308^{* *}$ & -0.009 & -0.063 & 0.087 & 0.024 & -0.074 & 0.044 & -0.051 & 0.080 & 0.135 & 0.044 \\
\hline $\mathbf{R E}$ & & & $0.344^{* *}$ & -0.010 & -0.021 & -0.094 & 0.050 & 0.071 & $0.215^{-}$ & -0.152 & -0.062 & -0.158 & -0.007 \\
\hline $\mathbf{A M}$ & & & & 0.054 & 0.089 & -0.020 & 0.038 & -0.005 & -0.126 & -0.004 & -0.121 & -0.051 & 0.063 \\
\hline IE & & & & & $\begin{array}{r}0.262^{*} \\
*\end{array}$ & 0.194 & $\begin{array}{r}0.292^{*} \\
*\end{array}$ & $\begin{array}{r}0.443^{*} \\
*\end{array}$ & $0.222^{*}$ & 0.123 & 0.116 & 0.120 & 0.046 \\
\hline PA & & & & & & $0.234^{*}$ & $\begin{array}{r}0.448^{*} \\
*\end{array}$ & $\begin{array}{r}0.333^{*} \\
*\end{array}$ & $0.218^{*}$ & $0.314^{* * *}$ & 0.176 & $\begin{array}{r}0.258^{*} \\
*\end{array}$ & 0.001 \\
\hline $\mathbf{R P}$ & & & & & & & 0.189 & 0.077 & 0.171 & $0.219^{*}$ & $0.245^{*}$ & 0.023 & 0.158 \\
\hline AS & & & & & & & & $\begin{array}{r}0.485^{*} \\
*\end{array}$ & 0.094 & $0.413^{* *}$ & $0.367^{* *}$ & $0.261_{*}^{*}$ & $\begin{array}{r}0.305^{*} \\
*\end{array}$ \\
\hline RC & & & & & & & & & 0.091 & $0.247^{*}$ & 0.135 & $0.218^{*}$ & -0.030 \\
\hline $\mathbf{O P}$ & & & & & & & & & & $0.370^{* * *}$ & $0.205^{*}$ & $\begin{array}{r}0.273^{*} \\
*\end{array}$ & 0.100 \\
\hline INE & & & & & & & & & & & $0.426^{* *}$ & $0.212^{*}$ & $0.271^{*}$ \\
\hline $\begin{array}{l}\text { AA } \\
\text { SD }\end{array}$ & & & & & & & & & & & & $0.239^{*}$ & $\begin{array}{l}0.186 \\
0.095\end{array}$ \\
\hline
\end{tabular}

**: $p<0.01 . * . p<0.05$.

IM: Intrinsic Motivation; IR: Identified Regulation; ER: External Regulation; AM: Amotivation; IE: Introvert-Extrovert; PA: Passive-Active; RC: Reserved-Communicative; RP: Reactive-Proactive; AS: Asocial-Sociable; OP: OptimisticPessimistic; INE: Indifferent-Empathetic; AA: Aggressive-Assertive; IA: Inflexible-Adaptive; SD: Submissive-Dominant. 
Regarding the "identified regulation", it correlates negatively and significantly with the "amotivation" ( $r=-0.308)$.

On the other hand, the "external regulation" correlates positively and significantly with the "amotivation" $(r=0.344)$. Likewise, it correlates significantly negatively with "optimism" $-\mathrm{OP}-(\mathrm{r}=$ $-, 215)$.

Finally, the "amotivation" (AM) correlates significantly in a negative way with the "intrinsic motivation" -MI- $(\mathrm{r}=-, 249)$ and with the "identified regulation" $(r=-0.308)$, as well as with most of social skills.

\section{DISCUSSION}

The main objective of this work is to develop an analysis of motivation in university students enrolled in the Nursing degree and compare with physical student's motivation.

As the data provided by the results demonstrate, the university population is mainly female (Senyuva et al., 2014), fact that is related to the sample in Physical Education Degree of CamposMesa et al. (2019), and contrasting with the results obtained in other degrees such as the degree in Physical Education (González-Valero et al., 2019), where the population is eminently male.

In this sense, this study reveals that the predominant motivation is intrinsic and that it is higher in women than men (Gutiérrez \& Tomás, 2018; Grunschel et al., 2016), contrasting with Campos-Mesa et al. (2019) study in physical education students, that showed a high level of intrinsic and extrinsic motivation, with statistically significant differences in extrinsic motivation.

Overall, the students of the nursing degree show a medium-high motivational level (Senol \& Akdag, 2018), while the physical education students show a high motivational level (Campos-Mesa et al., 2019). This fact is the materialization of the correspondence between the professional vocation of the student body and the degree.

On the other hand, the predominance of intrinsic motivation in university students is verified, with higher mean values in the feminine gender than in the masculine, as well as the existence of a negative relation between this and the amotivation, reason why the increase of one implies the decrease of the other and vice versa (Tasgin \& Coskun, 2018; Stenling et al., 2018).

Likewise, there is a significantly negative correlation, of a moderate nature, between intrinsic motivation and amotivation, so that an increase in one in students would imply a reduction in the opposite.

Regarding religion, although no statistically relevant differences have been identified, the highest mean values have been found in Christian/Catholic students. This reinforces what Lowicki et 
al. (2020), in whose study on empathy, religion and cognitive mentality capacity concluded that there was a great influence of religiosity on psychosocial variables such as empathy and motivation.

Interacting continuously, there is a direct relationship between motivation and student's social skills. Among the social skills that are related to motivation (and, therefore, influence each other) is the sociability of the student body, related to the intrinsic motivation and the identified regulation. This is attributed to the process of internalization of the motivational locus whereby a sociable person can start with an extrinsic motivation (social approval) and internalize itself (Ryan \& Deci, 2017).

On the other hand, there is a significantly positive relationship between intrinsic motivation and optimistic thinking. Likewise, external regulation correlates negatively significantly with optimism and positively with pessimism. Therefore, optimistic students have greater intrinsic motivation, while pessimistic students tend to externalize the motivational stimulus, that is, by feeling less socially competent, students tend to motivate themselves with external stimuli, while students who feel Socially competent, he believes more in himself and, therefore, his motivation is more self-determined. Shaw et al. (2018) relate this to greater social adaptability and better motivational and emotional regulation, while pessimism associates it with a greater sensitivity for negative affect along with a tendency to down-regulate positive affect.

Regarding emotional control, aggressiveness negatively correlates with intrinsic motivation in a negative way, while assertiveness maintains a significantly positive correlation. In this sense, aggressive students show very little intrinsic motivation, while assertive students have higher levels of intrinsic motivation (Rivera et al., 2019).

In this sense, authors such as Shi \& Du (2020), and Graf et al. (2020) exposes that the existence of a development in social skills implies an increase in other psychosocial factors, such as motivation, emotional intelligence, and communication skills; and when the level of these factors increases, the students experiment a greater social development.

Through this study, it has been revealed, on the one hand, that the student population of the Nursing degree is mainly female and comes from a Christian / Catholic religious culture, whose initial motivation to enroll in said degree was the professional vocation towards it.

On the other hand, students show a medium-high level of general social competence. In this sense, the social skills that the students declare to have the most developed are adaptability, empathy and sociability, while the least developed are thinking (pessimistic or optimistic) and social control (submission or domination).

Regarding motivation, the dimension with the highest score was the identified regulation, which highlights the intrinsic nature of student motivation, but not entirely, since this type of 
regulation continues to maintain a slight extrinsic component, although they showed a medium-high level of motivation.

On the other hand, there are significant gender differences in levels of motivation, with women being more intrinsically motivated than men. Likewise, in the opposite pole, men are significantly more amotivated than women, who show lower levels of amotivation than men.

Regarding religion, there are no relevant differences between the different religious cultures, although Christian / Catholic students have higher average levels of motivation and amotivation than Muslim students.

Regarding social skills and motivation, optimistic students are more motivated than pessimists; pessimists have higher levels of amotivation than those of the optimist.

On the other hand, students with a higher level of assertiveness show a higher level of intrinsic motivation than aggressive students.

Regarding motivation, there is a negative, and significantly moderate, relationship between intrinsic motivation and amotivation, so when the motivation increases, the amotivation decreases and vice versa.

Likewise, regarding the student's social skills, there is a positive relationship between social skills and intrinsic motivation, so the more social skills are developed, the higher the level of motivation the students show.

On the other hand, amotivation and social skills maintain a negative relationship, so the lack of social competence in students materializes in higher levels of amotivation, while a high level of social ability would imply a decrease in levels of amotivation.

\section{CONCLUSIONS}

Through this study, it has been revealed, on the one hand, that the student population of the Nursing degree is mainly female and comes from a Christian / Catholic religious culture, whose initial motivation to enroll in said degree was the professional vocation towards it.

On the other hand, students show a medium-high level of general social competence. In this sense, the social skills that the students declare to have the most developed are adaptability, empathy and sociability, while the least developed are thinking (pessimistic or optimistic) and social control (submission or domination).

Regarding motivation, the dimension with the highest score was the identified regulation, which highlights the intrinsic nature of student motivation, but not entirely, since this type of 
regulation continues to maintain a slight extrinsic component, although they showed a medium-high level of motivation.

On the other hand, there are significant gender differences in levels of motivation, with women being more intrinsically motivated than men. Likewise, in the opposite pole, men are significantly more amotivated than women, who show lower levels of amotivation than men.

Regarding religion, there are no relevant differences between the different religious cultures, although Christian / Catholic students have higher average levels of motivation and amotivation than Muslim students.

Regarding social skills and motivation, optimistic students are more motivated than pessimists; pessimists have higher levels of amotivation than those of the optimist.

On the other hand, students with a higher level of assertiveness show a higher level of intrinsic motivation than aggressive students.

Regarding motivation, there is a negative, and significantly moderate, relationship between intrinsic motivation and amotivation, so when the motivation increases, the amotivation decreases and vice versa.

Likewise, regarding the student's social skills, there is a positive relationship between social skills and intrinsic motivation, so the more social skills are developed, the higher the level of motivation the students show.

On the other hand, amotivation and social skills maintain a negative relationship, so the lack of social competence in students materializes in higher levels of amotivation, while a high level of social ability would imply a decrease in levels of amotivation.

Related to physical student's motivation, the nursing degree students have a medium-high level of motivation, and the physical education students have a high level of motivation. The motivation in nursing degree students is intrinsic. The physical education students have high level of intrinsic and extrinsic motivation.

The main limitation of this study has been the small samples that have been studied. This opens a new line of research, expanding both samples and comparing them.

This study has highlighted the importance of studying the motivation of students for a better educational coexistence and a greater adaptation of the teaching-learning process. 


\section{REFERENCES}

1. Bosman, R.; Roorda, D.; van der Veen, I.; \& Koomen, H. (2018). Teacher-student relationship quality from kindergarten to sixth grade and students' school adjustment: A person-centered approach. Journal of School Psychology., 68, 177-194. https://doi.org/10.1016/j.jsp.2018.03.006

2. Campos-Mesa, M., González-Campos, G., \& Castañeda-Vázquez, C. (2019). Análisis de la motivación del estudiante de educación superior participante en una propuesta de evaluación formativa. SPORT TK-Revista EuroAmericana De Ciencias Del Deporte, 8(2), 53-58. https://doi.org/10.6018/sportk.401111

3. Dörnyei, Z. (2018). Motivating Students and Teachers. The TESOL Encyclopedia of English Language Teaching, 1-6. https://doi.org/10.1002/9781118784235.eelt0128

4. Fernandez-Lasarte, O., Goñi, E., Camino, I., \& Zubeldia, M. (2018). Ajuste escolar y autoconcepto académico en la Educación Secundaria. Revista De Investigación Educativa, 37(1), 163-179. https://doi.org/10.6018/rie.37.1.308651

5. González-Valero, G.; Zurita-Ortega, F.; Pérez-Cortés, A.; Padial-Ruz, R.; Ubago-Jiménez, J.; \& Chacón-Cuberos, R. (2019). Estudio descriptivo del clima motivacional percibido hacia el deporte según el sexo de los futuros docentes de Educación Física. Sportis. Scientific Journal of School Sport, Physical Education and Psychomotricity, 5, 85-100.

6. Graf, J., Loda, T., Zipfel, S., Wosnik, A., Mohr, D., \& Herrmann-Werner, A. (2020). Communication skills of medical students: survey of self- and external perception in a longitudinally based trend study. BMC Medical Education, 20(1). https://doi.org/10.1186/s12909-020-02049-w

7. Grunschel, C., Schwinger, M., Steinmayr, R., \& Fries, S. (2016). Effects of using motivational regulation strategies on students' academic procrastination, academic performance, and well-being. Learning and Individual Differences, 49, 162-170. https://doi.org/10.1016/j.lindif.2016.06.008

8. Guay, F., Vallerand, R., \& Blanchard, C. (2000). Motivation and Emotion, 24(3), 175-213. https://doi.org/10.1023/a:1005614228250

9. Gutiérrez, M., \& Tomás, J. (2018). Clima motivacional en clase, motivación y éxito académico en estudiantes universitarios. Revista De Psicodidáctica, 23(2), 94-101. https://doi.org/10.1016/j.psicod.2018.02.001 
10. Kim, T., Kim, Y., \& Kim, J. (2018). Role of Resilience in (De)Motivation and Second Language Proficiency: Cases of Korean Elementary School Students. Journal of Psycholinguistic Research, 48(2), 371-389. https://doi.org/10.1007/s10936-018-9609-0

11. Łowicki, P., Zajenkowski, M., \& Van Cappellen, P. (2020). It's the heart that matters: The relationships among cognitive mentalizing ability, emotional empathy, and religiosity. Personality and Individual Differences, 161, 109976. doi: https://doi.org/10.1016/j.paid.2020.109976

12. Martín-Albo, J., Núñez, J., \& Navarro, J. (2009). Validation of the Spanish Version of the Situational Motivation Scale (EMSI) in the Educational Context. The Spanish Journal of Psychology, 12(2), 799-807. https://doi.org/10.1017/s113874160000216x

13. Meijer, E., Cleiren, M., Dusseldorp, E., Buurman, V., Hogervorst, R., \& Heiser, W. (2018). Cross-Validated Prediction of Academic Performance of First-Year University Students: Identifying Risk Factors in a Nonselective Environment. Educational Measurement: Issues and Practice, 38(1), 36-47. https://doi.org/10.1111/emip.12204

14. Rivera, J.; Lay, N.; Moreno, M. E.; Perez, A.; Rocha, G.; Parra, M.; Duran, S. E.; Garcia, J.; Redondo, O.; \& Torres-Rivas, E. (2019). Training program to develop social skills in university students. Espacios, 40, 1-15.

15. Ryan, R. M.; Deci, E. L. (2017). Self-determination theory: Basic psychological needs in motivation, development, and wellness, Guilford Press.

16. Şenol, C.; \& Akdağ, M. (2018). The Relation between Prospective Teachers' Attitudes towards Uncertainty and Motivation in Teaching. Education Sciences, 8, 122. https://doi.org/10.3390/educsci8030122

17. Şenyuva, E., Kaya, H., \& Bodur, G. (2014). Effect Social Skills of Nursing Students of the Project Based Teaching Methods. Procedia - Social and Behavioral Sciences, 152, 393-398. https://doi.org/10.1016/j.sbspro.2014.09.218

18. Shaw, D., Czekóová, K., Pennington, C., Qureshi, A., Špiláková, B., \& Salazar, M. et al. (2018). You $\neq$ me: individual differences in the structure of social cognition. Psychological Research, 84(4), 1139-1156. https://doi.org/10.1007/s00426-018-1107-3

19. Shi, M., \& Du, T. (2020). Associations of emotional intelligence and gratitude with empathy in medical students. BMC Medical Education, 20(1). https://doi.org/10.1186/s12909-020$\underline{02041-4}$

20. Stenling, A., Ivarsson, A., Lindwall, M., \& Gucciardi, D. F. (2018). Exploring longitudinal measurement invariance and the continuum hypothesis in the Swedish version of the 
Behavioral Regulation in Sport Questionnaire (BRSQ): An exploratory structural equation modeling approach. Psychology of Sport and Exercise, 36, 187-196. https://doi.org/10.1016/j.psychsport.2018.03.002

21. Tapia-Gutiérrez, C. P., \& Cubo-Delgado, S. (2017). Habilidades sociales relevantes: percepciones de múltiples actores educativos. Magis, Revista Internacional de Investigación en Educación, 9(19), 133-148. https://doi.org/10.11144/javeriana.m9-19.hsrp

22. Tasgin, A., \& Coskun, G. (2018). The Relationship between Academic Motivations and University Students' Attitudes towards Learning. International Journal of Instruction, 11(4), 935-950. https://doi.org/10.12973/iji.2018.11459a

23. Van der Stouwe, T., Asscher, J. J., Hoeve, M., van der Laan, P. H., \& Stams, G. J. J. M. (2016). The Influence of Treatment Motivation on Outcomes of Social Skills Training for Juvenile Delinquents. International Journal of Offender Therapy and Comparative Criminology, 62(1), 108-128. https://doi.org/10.1177/0306624x16648130

\section{AUTHOR CONTRIBUTIONS}

All authors listed have made a substantial, direct and intellectual contribution to the work, and approved it for publication.

\section{CONFLICTS OF INTEREST}

The authors declare no conflict of interest.

\section{FUNDING}

This research received no external funding.

\section{COPYRIGHT}

(C) Copyright 2022: Publication Service of the University of Murcia, Murcia, Spain. 